\title{
Determination of particle size by using the angular distribution of backscattered light as measured with low-coherence interferometry
}

\author{
Adam Wax, Changhuei Yang, Vadim Backman, Maxim Kalashnikov, Ramachandra R. Dasari, \\ and Michael S. Feld \\ G. R. Harrison Spectroscopy Laboratory, Massachusetts Institute of Technology, 77 Massachusetts Avenue, \\ Cambridge, Massachusetts, 02139
}

Received May 25, 2001; revised manuscript received August 2, 2001; accepted September 26, 2001

\begin{abstract}
We employ a novel interferometer to measure the angular distribution of light backscattered by a turbid medium. Through comparison of the measured data with the predictions of Mie theory, we are able to determine the size of the scatterers comprising the medium with subwavelength precision. As the technique is based on low-coherence interferometry, we are able to examine the evolution of the angular distribution of scattered light as it propagates into the medium. The effects of multiple scattering as a function of penetration depth in the medium are analyzed. We also present various considerations for extending this technique to determining structural information in biological tissues, such as the effects of a distribution of particle sizes and the need to average out speckle contributions. (c) 2002 Optical Society of America
\end{abstract}

OCIS codes: $290.3200,170.4500$.

\section{INTRODUCTION}

The structure of light-scattering objects can be investigated by examining their angular scattering characteristics. Angle-resolved light-scattering distributions are conventionally obtained by using a goniometer ${ }^{1}$ but can also be measured by interferometric techniques. ${ }^{2}$ Recently we have developed a modified Michelson interferometer that is capable of measuring the angular distribution of backscattered light. ${ }^{3}$ This technique is implemented by using a low-coherence source, which permits depth resolution in the same way as optical coherence tomography. ${ }^{4}$

Recent interest in the use of light-scattering techniques to measure structural properties has focused on measuring the size of cell nuclei and other subcellular organelles within biological tissues. In particular, light-scattering spectroscopy has shown that cell nuclei can be regarded as spherical Mie objects and their size determined by comparing the wavelength dependence of their backscattering cross section with theoretical models. ${ }^{5}$ This technique has been demonstrated to be an effective means in detecting irregularities in the structure of epithelial cell nuclei associated with neoplasia, a precancerous state. ${ }^{6}$

Our new technique seeks to create a synthesis between optical coherence tomography and light-scattering spectroscopy. The former technique is capable of producing tomographic images of the microscopic structure of biological tissues, but conventional implementations lack the resolution for quantitative assessment of changes in nuclear size. In contrast, the latter technique can accurately determine the size of cell nuclei but lacks depth resolution, relying on polarization gating or modeling to remove multiply scattered photons. Early efforts to combine these two methods sought to include multiple wave- lengths of broadband light in a Michelson interferometer. ${ }^{7}$ Instead, we now take a different approach and seek to recover structural information by examining the angular distribution of backscattered light with the use of a single broadband light source.

Our current technique employs ideas developed with previous interferometric techniques for measuring the optical phase-space distribution of a light field, ${ }^{8}$ i.e., its joint position and momentum distribution. The properties of the field of interest, in our case the light backscattered by a sample, can be mapped out by mixing the field with a reference field with variable properties. While measurement of the phase-space distribution of a light field is a powerful tool for examining the propagation of optical coherence in multiple-scattering media, ${ }^{9,10}$ we will show that the measurement of angular distributions alone is sufficient for the task of determining the size of a scattering object in a surface layer. In addition, the current technique offers the advantage that it is implemented in a Michelson geometry and thus is compatible with optical coherence tomography.

In this paper we describe measurements of the angular distributions of light backscattered by turbid media consisting of polystyrene microsphere suspensions. We demonstrate that structural information can be determined with subwavelength precision from the surface layer by comparing the measured angular distributions with the predictions of Mie theory. Since the refractive index of the polystyrene microspheres is well characterized, the current work focuses only on determining their size; however, comparisons of experimental data with Mie theory predictions could also be made to determine refractive index. We discuss the experimental and modeling issues that affect determination of the size of a scatterer from an angular distribution of scattered light, such as the need 
for ensemble averaging to overcome speckle and the effects of multiple scattering. Finally, we present some of the considerations for extending this approach to determining structure in biological tissues where nonspherical shapes, variations in particle size, and speckle can hinder the modeling of scatterers as Mie particles.

The remainder of this paper is organized as follows. The modified Michelson interferometer is described in Section 2. We use this method to measure the angular distribution of light backscattered by suspensions of polystyrene microspheres. These measurements are also presented in Section 2. The analysis of the data, which permits determination of the size of the scatterers, is presented in Section 3. Finally, a discussion of the considerations for extending this approach to biological tissues is presented in Section 4.

\section{EXPERIMENT}

We have developed a low-coherence interferometry scheme that allows quick and simple measurement of the angular distribution of light backscattered by an arbitrary sample. We apply this technique to measure the angular distribution of light backscattered by suspensions of polystyrene microspheres with various physical properties.

\section{A. Scheme}

The low-coherence interferometry scheme diagrammed in Fig. 1 and used to measure angular scattering distributions in these experiments is a modified version of our earlier method. ${ }^{3}$ The modifications permit measurements to be made over a broader range of angles, extending the previous range of -120 to $+120 \mathrm{mrad}$ to a new range of 0-480 mrad (approximately $27.5 \mathrm{deg}$ ). The ability to measure light scattering at larger angles improves the accuracy and range for determining particle sizes. Further, in the new scheme, we vary the length of the reference arm rather than moving the sample to generate a Doppler shift.

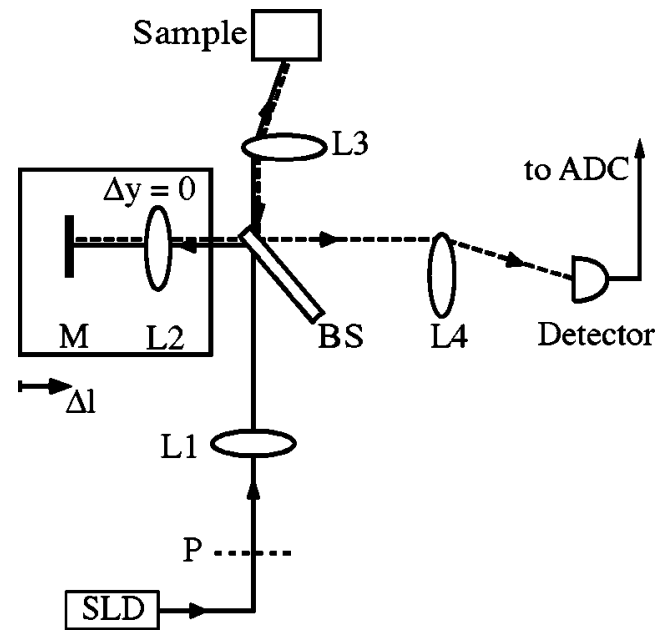

Fig. 1. Schematic of the interferometer system illustrating the detection of backscattered light. The photocurrent is digitized by an analog-to-digital converter, $\mathrm{ADC}$, and recorded on a personal computer.
The light source is a superluminescent diode purchased from EG\&G, Inc., with an output power of $3 \mathrm{~mW}$, a center wavelength of $845 \mathrm{~nm}$ and a FWHM bandwidth of $22 \mathrm{~nm}$, resulting in a coherence length, $l_{c}$, of $2 \ln 2 / \pi \times \lambda^{2} / \Delta \lambda$ $=14.3 \mu \mathrm{m}$. As in conventional low-coherence interferometery, the light from the source is split by a beam splitter BS into a reference beam and an input beam to the sample. The reference beam is reflected by a mirror M and recombined at BS with light reflected by the sample. The mixed fields generate an interference pattern provided that the two optical path lengths are matched to within the coherence length of the source. In order to separate the interference signal from the dc background and low frequency noise, we generate a heterodyne signal by translating the reference mirror $M$ at a constant velocity of $4 \mathrm{~mm} / \mathrm{s}$. This causes the heterodyne beat intensity to oscillate at the corresponding Doppler shift frequency of $9.5 \mathrm{kHz}$. The beat intensity is determined by calculating the power spectrum of the digitized photocurrent sampled at $333 \mathrm{kHz}$ and bandpassing the signal at the heterodyne frequency. This yields a signal that is linearly proportional to the squared amplitude of the detected signal field. ${ }^{11}$ The data presented in this paper represent the square root of this quantity.

The interferometer relies on four achromatic imaging lenses L1-L4 to permit the measurement of angular scattering distributions. Lenses L1 and L2 are arranged to form a modified $4 f$-imaging system. In this system, the distance from the input plane $\mathrm{P}$ to the first lens $\mathrm{L} 1$ is equal to the focal length of the lens, $f_{1}=10 \mathrm{~cm}$. The distance between lenses L1 and L2 is the sum of their focal lengths, $f_{1}+f_{2}$, and the output plane is a distance $f_{2}=4.5 \mathrm{~cm}$ beyond lens L2. This arrangement produces at the output plane-in this case reference mirror $\mathrm{M}-\mathrm{a}$ scaled version of the field at the input plane $P$. The reproduced spatial distribution is scaled by a factor of $f_{2} / f_{1}=0.45$, while the associated angular distribution is scaled larger by $f_{1} / f_{2}=2.22$. The modified $4 f$-imaging system composed of lenses $\mathrm{L} 1$ and $\mathrm{L} 3\left(f_{3}=4.5 \mathrm{~cm}\right)$ is responsible for imaging the field at plane $\mathrm{P}$ onto the input face of the sample. This imaging system is different from that comprising lenses L1 and L2 in that lens L3 is laterally displaced relative to lens L1. This translation results in the input beam entering the sample at an angle of $230 \mathrm{mrad}$. In addition, this arrangement permits the use of the full angular aperture of the lens to be used to collect angular backscattering data, rather than only the halfangle that would be possible with normal incidence. Aside from the linearly varying phase that accounts for the change in the angle of propagation, a scaled replica of the phase and amplitude of the field at plane $\mathrm{P}$ with features similar to those produced at the reference mirror $\mathrm{M}$ by lenses L1 and L2 is produced at the focal plane of lens L3 $\left(f_{3}=4.5 \mathrm{~cm}\right)$, which coincides with the input face of the sample. This arrangement permits selection of the form of both the reference field and the field used to probe the sample simply by inserting a lens at the output of the superluminescent diode to shape the beam to the desired size and angular divergence at plane $\mathrm{P}$.

Additional $4 f$-imaging systems, constituted by lenses L2 and L4 (reference) and L3 and L4 (signal), are also used to reproduce the phase and amplitude of the scat- 


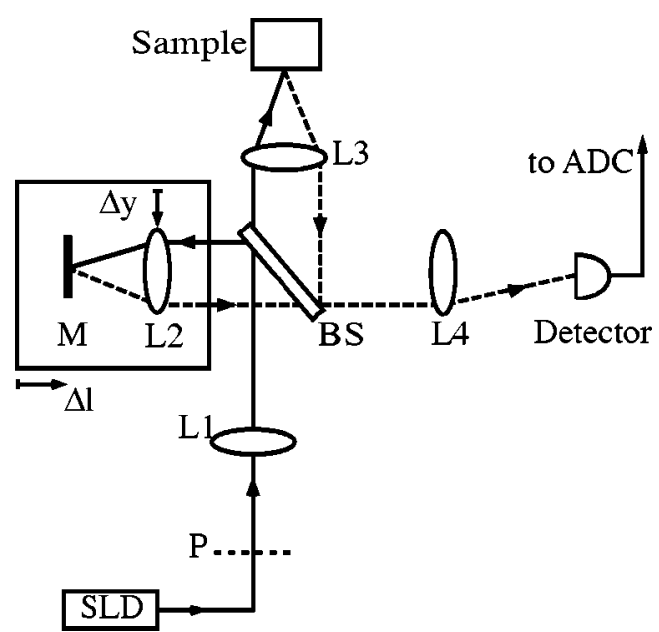

Fig. 2. Schematic of the interferometer system illustrating the displacement of lens L2 to vary the angle at which the reference beam crosses the detector plane.

tered field and the reference field in the plane of the detector. Since lens L4 has focal length $f_{4}=10 \mathrm{~cm}$, the spatial and angular distributions are again rescaled according to the ratio of the focal lengths. Analysis of the imaging system with Fourier optics shows that by scanning lens L2 a distance $\Delta y$ perpendicular to the beam path, the reference field is reproduced in the plane of the detector with its angle of propagation changed to $\theta_{T}$ $=2 \Delta y / f_{2}$ but its position unchanged. ${ }^{12}$ The path length of the light through the system remains unchanged as the lens is translated transversely to the beam path. To preserve this property of the imaging system, the lens L2 is moved in conjunction with the reference mirror $\mathrm{M}$ along the direction of the beam path while the length of the reference arm is varied. This does result in small changes to the wave-front curvature of the reference beam as it crosses the detector plane. However, since the length of the reference arm is varied by a small amount $(<1 \mathrm{~mm})$ compared with the Rayleigh length of the beam $(\sim 1 \mathrm{~mm})$, the effect is negligible. Figure 2 illustrates the effects of transversely scanning lens L2 in the imaging system by using ray traces. It can be seen that light is now collected at a specific angle relative to the backscattering direction.

In the current scheme, the maximum clear aperture limits the angular scans to a total range of $480 \mathrm{mrad}$. The angular resolution, $\theta_{\text {res }}=1.4 \mathrm{mrad}$, is given by the diffraction angle of the $0.45-\mathrm{mm}$-diameter collimated beam at the sample, corresponding to a 1-mm-diameter beam input to the interferometer at the plane P. Since the angular distributions we seek to measure are symmetric about the backscattering direction, the system is implemented such that the scan begins in the backscattering direction and extends $480 \mathrm{mrad}$ away from that direction. As discussed above, the light enters the sample at an angle of $240 \mathrm{mrad}$ relative to the sample surface. However, for a sample containing particles suspended in fluid, the backscattering direction will still be antiparallel to the input beam, regardless of the orientation of the sample surface.

\section{B. Data}

We have measured the angular distribution of light backscattered by a turbid medium consisting of polystyrene microspheres ( $n=1.59$ ) suspended in a mixture of $20 \%$ glycerol and $80 \%$ water. The mixture $(n=1.36)$ is chosen to provide neutral buoyancy for the microspheres; i.e., the specific gravity of the mixture matches that of the microspheres. The relative refractive-index difference between the microspheres and the medium is $n_{\text {rel }}=1.17$.

The microspheres are chosen to be comparable in size to epithelial cell nuclei, and the concentration is chosen to produce a mean free path comparable to that found in epithelial tissues. The microspheres (Bangs Laboratories, Inc.) are specified by the manufacturer to have a 10.06- $\mu \mathrm{m}$ diameter with a standard deviation of $0.1 \mu \mathrm{m}$. Using Mie theory, we calculate the scattering cross section $\sigma_{S}$ to be 2.45 times the geometrical cross section, yielding $\sigma_{S}=194 \mu \mathrm{m}^{2}$. The concentration of the microspheres, $\rho=5.14 \times 10^{10} / \mathrm{cm}^{3}$, is chosen to yield a mean free path of $l_{S}=1 / \rho \sigma_{S}=100 \mu \mathrm{m}$.

Figure 3 shows the angular distribution of light backscattered from this sample as a function of the penetration depth into the sample. The angle is given in radians, and the penetration depth is given in units of optical depth $\tau=l / l_{S}$, where $l$ is the optical path length in the medium. This figure shows six angular lobes of higher field amplitude for light scattered near the surface $(\tau=0)$. Figure 4 shows a plot of the angular distribution for $\tau=0.5$. As the light penetrates deeper into the medium, the contrast of the lobes begins to fade. (Fig. 5, $\tau=3.5$ ), until only the central lobe near the backscattering direction is present (Fig. 6, $\tau=5.5$ ).

The measured angular distribution for scattering from the surface layer (Fig. 4) is compared with the predictions of Mie theory. The determination of particle size by such comparison is discussed below in Subsection 3.A. The scattering from subsurface layers (Figs. 5 and 6) is com-

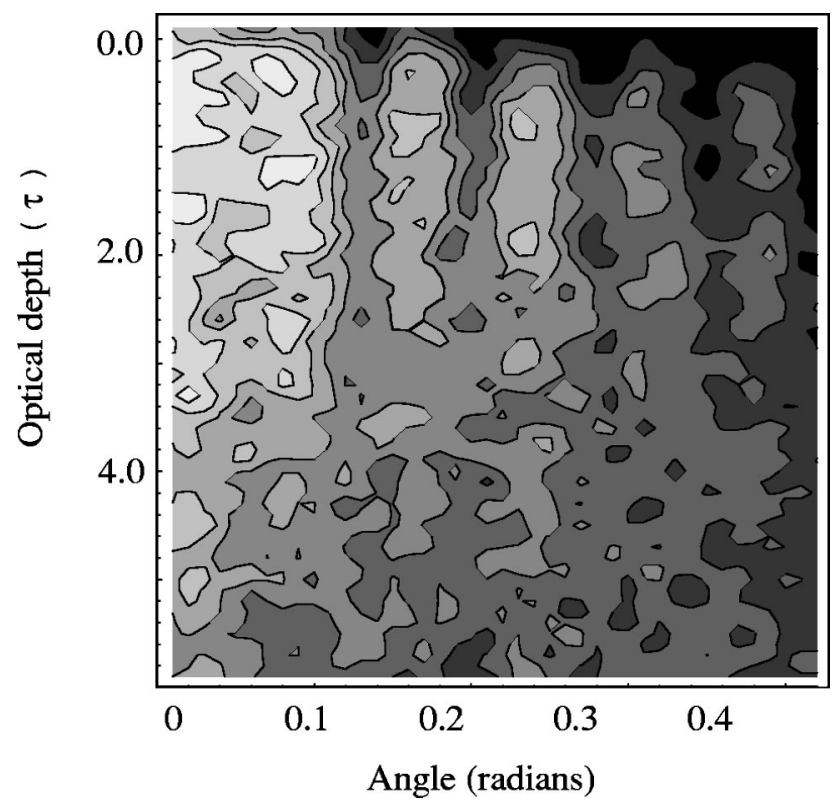

Fig. 3. Contour map of the measured angular distribution of light backscattered by turbid media as a function of penetration depth. White areas represent $-4.6 \mathrm{~dB}$; black, $-7 \mathrm{~dB}$. 


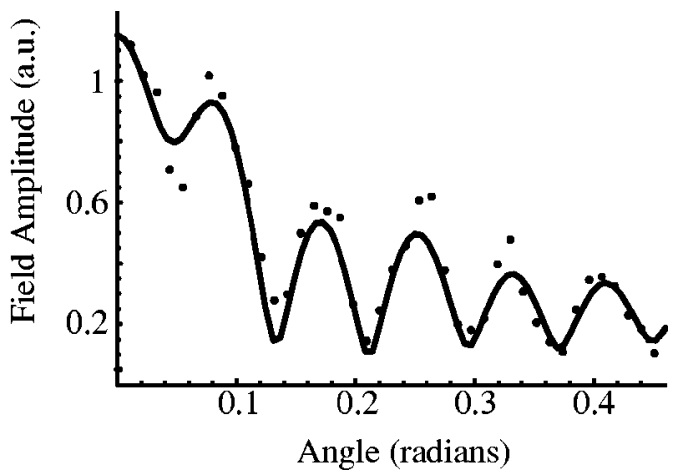

Fig. 4. Angular distribution of light backscattered from a surface layer $\quad \tau=0.5$. Dots represent experimental data; solid curve, theoretical distribution.

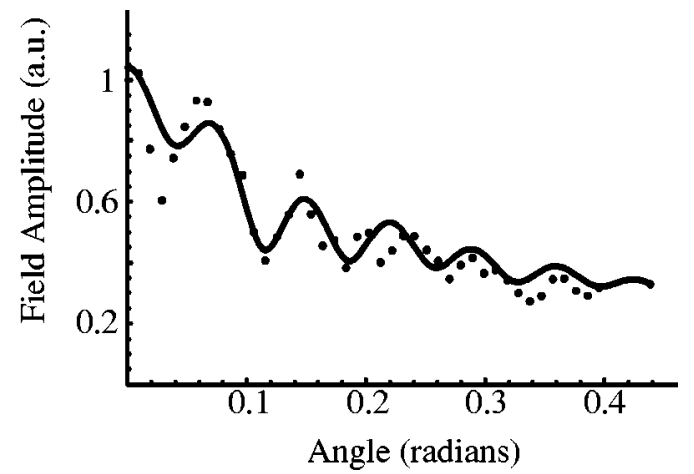

Fig. 5. Angular distribution of light backscattered from a subsurface layer $\tau=3.5$. Dots represent experimental data; solid curve, theoretical distribution.

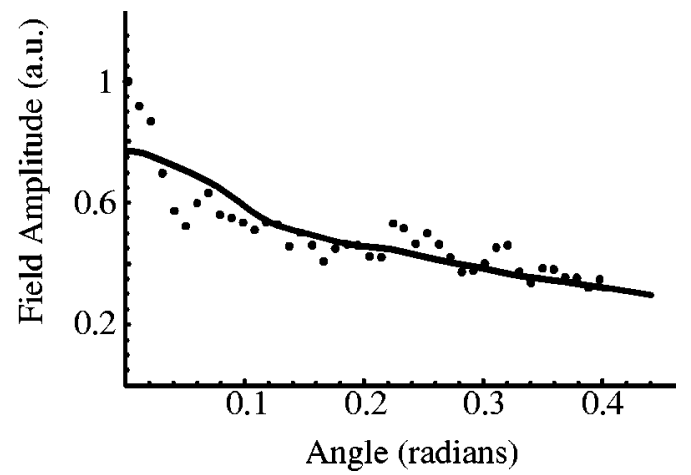

Fig. 6. Angular distribution of light backscattered from a subsurface layer $\tau=5.5$. Dots represent experimental data; solid curve, theoretical distribution.

pared with theoretical models of the contribution of multiple scattering. A description of these models is given below in Subsection 3.B.

\section{DATA ANALYSIS}

\section{A. Comparison with Mie Theory}

The fit to the data shown in Fig. 4 is obtained by comparing the measured angular distribution with a series of theoretical distributions for various sizes and distributions of sizes of particles. The theoretical distributions are calculated from Mie theory for a range of particle diameters (1.0 $\mu \mathrm{m}$ to $15.0 \mu \mathrm{m}$ in $0.1-\mathrm{mm}$ steps), as well as various distributions of particle size (1/e width of the Gaussian distribution is $1.0 \%, 2.5 \%, 5.0 \%, 7.5 \%, 10 \%$, $15 \%$ and $20 \%$ of the mean diameter), and the results are stored in a lookup table. The best fit to the data is found by computing the chi-squared value for each distribution and finding the minimum value. Figure 7 shows the chisquared values for comparisons of the measured data from Fig. 4 to theoretical angular distributions for a range of scatterer sizes and a fixed relative refractive index ( $n=1.17)$ and distribution of sizes ( $1 / e$ width is $2.5 \%$ of mean diameter, henceforth denoted as a $2.5 \%$ variation). Since the relative refractive index for the microspheres is well known, this parameter is not varied in optimizing the fit to the data. However, various size distributions are examined in the analysis of the data. While the chisquared value is minimized for a $2.5 \%$ variation in particle sizes, there is very little difference between it and the values obtained for the other distributions $(1 \%, 5 \%)$ used in the analysis. The $2.5 \%$ variation of the size distribution is larger than that specified by the manufacturer (1\%) but is a reasonable value when the broad bandwidth of the light is taken into consideration. This issue is discussed below in more detail in Subsection 4.A.

In Fig. 7 we see that the chi-squared values are parabolic near the 10- $\mu \mathrm{m}$ range of particle sizes. By fitting the distribution of chi-squared values in this range to a parabola, we find that the minimum value is 0.13 , corresponding to a particle size of $10.12 \pm 0.30 \mu \mathrm{m}$. The uncertainty in the fit is estimated by calculating the particle size for which the chi-squared value is twice the minimum value. For comparison, light-scattering spectroscopy techniques ${ }^{5}$ were used to find the size and size distribution of the same sample, yielding a mean size of $10.05 \mu \mathrm{m}$ and a size distribution of $0.5 \%$. Thus we are able to determine the average particle size in the medium with better-than-wavelength precision and accuracy.

\section{B. Multiple Scattering}

The experimental data shown in Fig. 3 show the evolution of the angular distribution as a function of penetration depth into the medium. In Fig. 4, the experimental distribution for the surface scattering is compared with the prediction of Mie theory. However, in Figs. 5 and 6, the experimental distributions depart from this prediction, and thus further analysis is needed to understand their features.

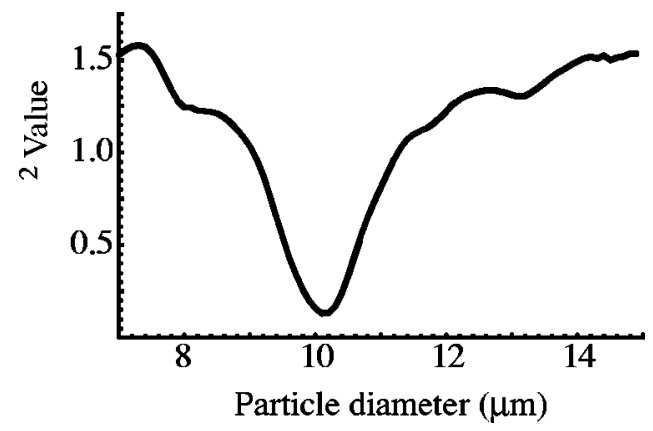

Fig. 7. Graph of chi-squared values between experimental data (Fig. 4) and angular distributions for various size spheres calculated by Mie theory. 


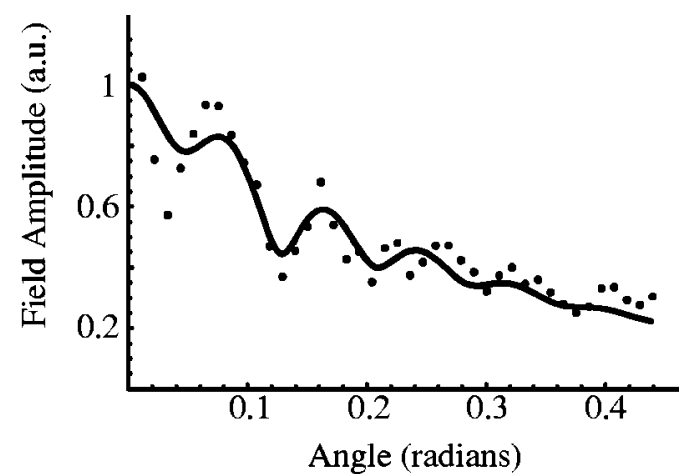

(a)

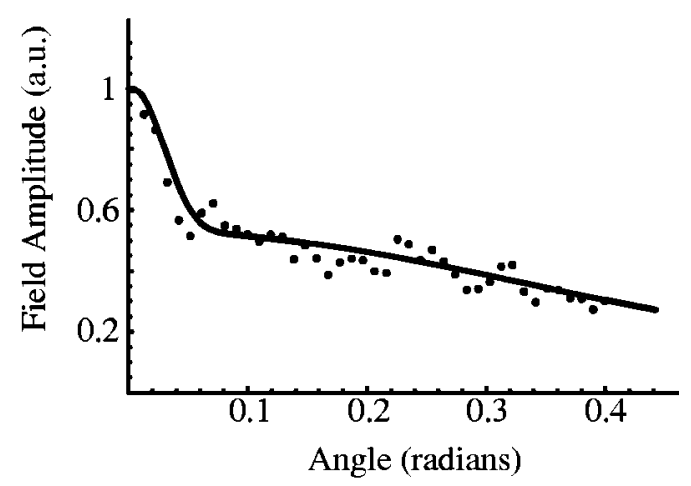

(b)

Fig. 8. Angular distribution of light backscattered from subsurface layers: (a) for $\tau=3.5$; (b) for $\tau=5.5$. Dots represent experimental data; solid curve, theoretical distributions based on scattering by polydisperse turbid media.

In Figs. 5 and 6, data are presented for light that has traveled $\tau=3.5$ and $\tau=5.5$ mean free paths in the medium, respectively. Qualitatively, we can see that the single-scattering theoretical distribution will not adequately describe these data. Instead, we see the presence of both single-scattering and multiple-scattering contributions. At angles near the backscattering direction, sharp oscillations characteristic of single scattering are seen. At larger angles, a broad distribution typical of multiple scattering is seen.

The theoretical distributions shown in Figs. 5 and 6 are constructed from linear combinations of the prediction for single scattering and an angular distribution predicted for two scattering events. The distribution for two scattering events is obtained by convoluting the angular distribution for scattering in the forward direction with that for the backward direction. Thus this distribution is intended to model the angular distribution of photons that have forward scattered once in addition to scattering once in the backward direction. The linear combination of the two theoretical distributions does not fit the data shown in Figs. 5 and 6 particularly well. It appears that the fit is a compromise between fitting the sharp features at small angles and the broad distribution seen at larger angles. Instead of using a linear combination of these two forms to fit the data, a treatment is needed that is able to discriminate the contribution of each form to the scattering seen at each angle. One possible approach is to use a wave-mechanical treatment that examines the optical phase-space (position-momentum) distribution such as those that have been applied to successfully model other scattering problems. ${ }^{9,10}$

Using an empirical approach, we model the multiplescattering distribution as a single scattering by a broad distribution of particle sizes in the medium. This approach yields theoretical distributions that more closely resemble the angular distributions seen in the experimental data. Figure 8(a) [8(b)] shows the experimental angular distribution for $\tau=3.5$ (5.5) compared with the theoretical distribution predicted by Mie theory for a $10 \%$ (25\%) distribution, of particle sizes. This approach yields better fits than the linear combination model described above, by providing theoretical distributions that contain both broad and sharp features.

\section{DISCUSSION: CONSIDERATIONS FOR APPLICATION TO BIOLOGICAL TISSUES}

There are two key considerations that must be addressed in order to apply this method of size determination to biological tissues. First, in the experiments described above, a fairly, monodisperse distribution of particle sizes is used. Although cell nuclei have been shown to behave as Mie scatterers, ${ }^{5}$ it is expected that they will have a broader distribution of sizes than the scatters used in the turbid medium in these experiments. Second, the experiments described here exploit Brownian motion to ensemble-average out the effects of speckle, i.e., the random interferences arising from particle-particle correlations. Biological tissue typically does not exhibit such motions, and therefore another means for overcoming speckle must be devised.

\section{A. Broad Range in Size Parameters}

In order to investigate the effects of polydisperse distributions of scatterer sizes, we measured the angular scattering distribution from a sample of polystyrene microspheres specified by the manufacturer (Duke Scientific, Inc.) to have a $20 \%$ variation in scatterer sizes. The angular distribution for the light scattered by the surface layer of this sample is shown in Fig. 9. The distribution shows only a single lobe in the backscattering direction with a broad feature extending over the remaining angular scan. This scan is similar to the structure of the scan

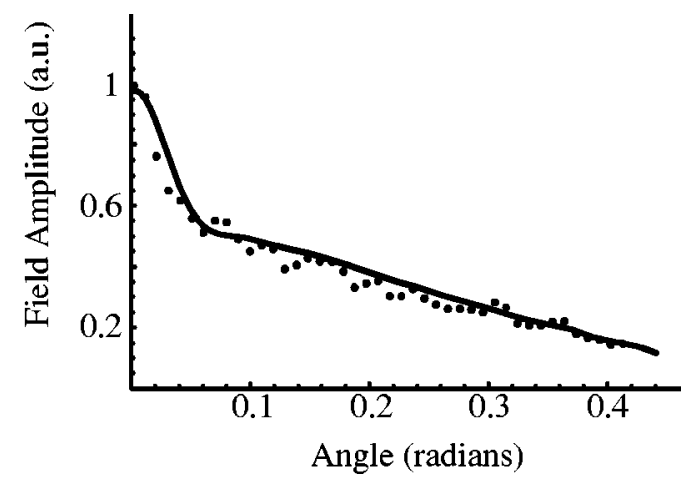

Fig. 9. Angular distribution of light backscattered from a surface layer of a polydisperse scattering medium. Dots represent experimental data; solid curve, theoretical distribution. 
from a subsurface layer shown in Figs. 6 and 8(b). In this case, comparison with Mie theory does not achieve the same high degree of precision obtained with the scan of the sample with a narrow size distribution. However, useful size information can still be recovered. We compare the measured angular distribution with theoretical angular distributions for several sizes and size distributions and compute the chi-squared value for each. We find that the chi-squared value is minimized for a size of $9.5 \mu \mathrm{m} \pm 2.7 \mu \mathrm{m}$ and a size distribution with a $25 \%$ variation in diameter, which is in reasonable agreement with the manufacturer's specifications. Thus we are able to determine particle size even for samples with a broad distribution of scatterer sizes.

A more direct approach is simply to examine the characteristics of the central lobe. Taking the view that there are two scattering processes that contribute to the overall scattering-diffraction and the combination of reflection and refraction-we can attribute this central lobe to the diffractive contribution to the scattering. ${ }^{12}$ The portion of the scattering that is due to reflection and refraction exhibits lobes that vary in magnitude and angular location with variations in size and refractive index. Thus a theoretical scattering distribution that averages many of these contributions, such as one would obtain for a medium with a broad distribution of scatterer sizes, will produce broad features. Conversely, the contribution that arises from diffraction is always near the backward and forward directions. Thus, averaging these contributions for many sizes of scatterers retains the narrow features in the forward and backward directions.

Information about the size of the particles can be obtained by fitting the diffractive component of the angular backscattering distribution to a Gaussian distribution. Using this approach, we find that the central lobe has a $1 / e$ width of $27.9 \mathrm{mrad}$. If we assume this width to be equal to $k a$, where $k$ is the wave number and $a$ is the radius, the particle diameter of $9.5 \mu \mathrm{m}$ is again recovered. This demonstrates further that useful size information can be recovered from a turbid medium with a broad distribution of particle sizes when this method is used.

An additional consideration that arises in calculating angular scattering patterns for low-coherence light by Mie theory or approximate methods is the interplay between the broad distribution of wavelengths and the distribution of scatterer sizes. Each factor will contribute to an overall broadening of the distribution of the size parameter $x=k a$. If we assume that both the wavelengths and sizes are Gaussian distributed, then the effect is a Gaussian distribution of size parameters with a width given as $(\Delta x / x)^{2}=(\Delta k / k)^{2}+(\Delta a / a)^{2}$, where $\Delta x$ is the 1/e width of the Gaussian distribution. This result is obtained by simply convolving the distribution in wavelengths with the distribution in sizes. This is an appropriate model since each wavelength component will interact with each size in the size distribution. In our experiments the bandwidth and the center wavelength produce a distribution of size parameters with a $1.6 \%$ variation. Thus we will be unable to measure size distributions with smaller variations than this.

In a previous experiment, the disagreement between measured angular distributions and monochromatic Mie theory was attributed to the coherence length of the broad bandwidth source. ${ }^{3}$ The agreement between data and theory during probing of a similar sample is then apparently restored by increasing the coherence length of the source through use of an optical filter to reduce the bandwidth. However, using the approach described above, the effects of a broadband source on the angular scattering distribution can be modeled by Mie theory. It is evident from the data presented below in Subsection 4.B. that the bandwidth of our source, which results in a $1.6 \%$ variation in the size parameters, cannot be responsible for the decrease in scattering at angles beyond the central backscattering lobe for 6.1- $\mu \mathrm{m}$-diameter microspheres. From the analysis presented above, one can see that to measure only the central backscattering lobe, a variation of size parameters near $20 \%$ is needed. This variation can arise from a combination of source bandwidth and distribution of particle sizes. Most likely, the discrepancy seen in Ref. 3 is due to a contaminated sample containing a wide distribution of particle sizes.

\section{B. Ensemble Averaging}

The data presented above are obtained by averaging five depth scans at each scattering angle. This serves to ensemble-average the light scattering by the sample to eliminate the effects of speckle. In the case of polystyrene microspheres suspended in fluid, the Brownian motion of the microspheres causes them to change positions between scans, effectively randomizing the phase of the light scattered by each microsphere. Upon averaging the multiple scans, the interference between light scattered by different microspheres averages to zero owing to this randomization of their relative phases. The number of scans at each scattering angle was chosen by examining the average of the multiple scans and determining a threshold beyond which no appreciable changes appeared with further averaging.

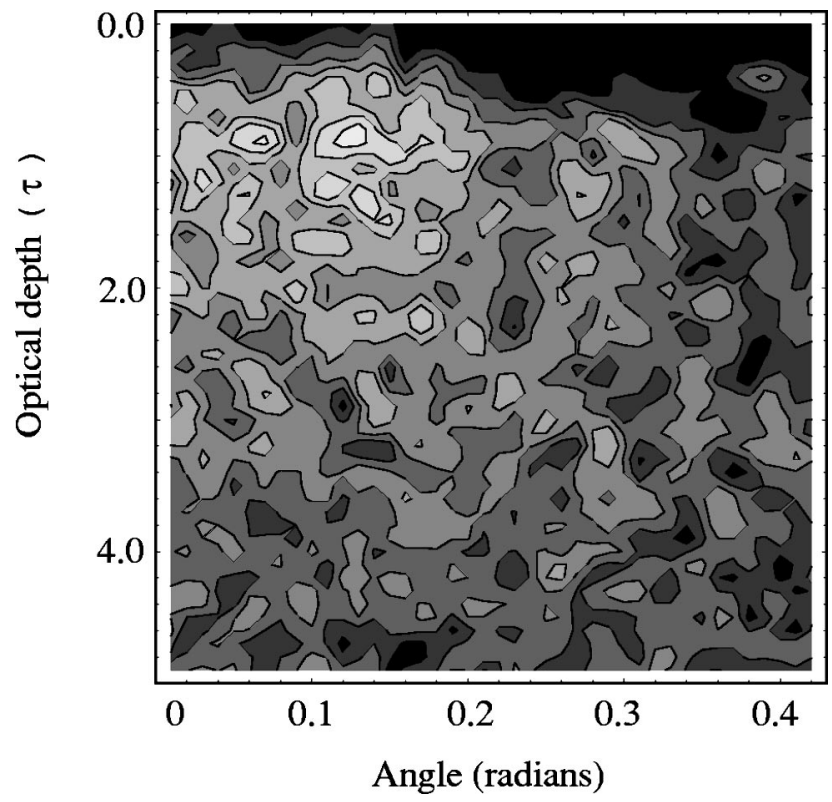

Fig. 10. Contour map of the measured angular distribution of light backscattered by turbid media as a function of penetration depth obtained without the benefit of ensemble averaging. White areas represent $-3.8 \mathrm{~dB}$; black, $-7 \mathrm{~dB}$. 


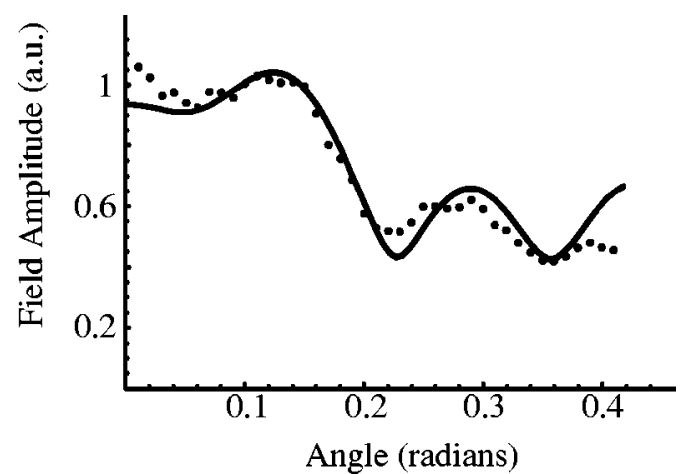

Fig. 11. Angular distribution of light backscattered by turbid media obtained by ensemble averaging using the scans from various depths. Dots represent experimental data; solid curve, distribution.

In situations where Brownian motion is not present, averaging many depth scans will not eliminate the effects of speckle, as in the case of cell nuclei in biological tissues. To achieve an ensemble average in this situation, one can use the average of the scattering from adjacent depths instead. To illustrate this point, we measured the angular distribution of light scattered by a turbid medium but did not use multiple depth scans to ensemble average. In this experiment the turbid medium contained $6.1-\mu \mathrm{m}$ microspheres (Polysciences, Inc.) at a concentration corresponding to a mean free scattering path of $100 \mu \mathrm{m}$. Figure 10 shows a contour map of the angular scattering distribution as a function of depth. It can be seen that these data suffer from increased noise in comparison with the data of Fig. 3.

We can achieve a reduction of noise due to speckle by averaging the angular scans from different depths. Figure 11 shows the angular scan resulting from averaging the angular scans from the top $100 \mu \mathrm{m}$ of the medium (ten angular scans). We compare this result with the predictions of Mie theory in the manner described above to determine that this medium is composed of microspheres of diameter $5.4 \mu \mathrm{m} \pm 0.4 \mu \mathrm{m}$ of a size distribution with a $2.5 \%$ variation. These values differ from those specified by the manufacturer, but these parameters have been confirmed with light-scattering spectroscopy, ${ }^{5}$ yielding a mean size of $5.5 \mu \mathrm{m}$ and a distribution with a $1 \%$ variation. Thus noise due to speckle can be reduced to acceptable levels through averaging of angular scans from different depths, permitting size determination by comparison with Mie theory.

\section{CONCLUSIONS}

In conclusion, we have shown that the size of scatterers in a turbid medium can be determined with subwavelength precision by measurement of angular scattering distributions with low-coherence interferometry. This method can easily be extended to determine their refractive index as well. The advantage of using low-coherence interferometry is that the measured angular distributions are depth resolved. This permits isolation of singly backscattered light from the surface layer. Examination of the angular distribution of light scattered from subsurface layers yields insight into the contribution of multiple scattering to low-coherence interferometry signals. The method described above has potential application to determining the size of cell nuclei in biological tissues, as is done with light-scattering spectroscopy. ${ }^{5,6}$ Conventional light-scattering spectroscopy examines wavelengthdependent scattering characteristics to make a size determination and uses either modeling or polarization subtraction to reject multiply scattered light. The lowcoherence-interferometry sizing method offers the advantage of using a single broadband light source and coherence gating to suppress multiply scattered light. We have presented some of the considerations that must be addressed for application of this method to biological tissues. Experiments are underway in our laboratory to use angular distributions measured with low-coherence interferometry to determine the size of structures in biological tissues.

\section{ACKNOWLEDGMENTS}

This work was conducted at the MIT Laser Biomedical Research Center and was supported by grants from the Hamamatsu Corporation, the National Science Foundation (CHE-0111370), and the National Institutes of Health (NIH) through the National Center for Research Resources (P41-RR02594). Adam Wax is supported by National Research Service Award fellowship grant from the NIH (F32 RR05075).

Author Adam Wax receives e-mail at awax@mit.edu.

\section{REFERENCES AND NOTES}

1. R. Drezek, A. Dunn, and R. Richards-Kortum, "Light scattering from cells: finite-difference time-domain simulations and goniometric measurements," Appl. Opt. 38, 36513661 (1999).

2. C. Yang, K. An, L. T. Perelman, R. R. Dasari, and M. S. Feld, "Spatial coherence of forward-scattered light in a turbid medium," J. Opt. Soc. Am. A 16, 866-871 (1999).

3. A. Wax, C. Yang, R. R. Dasari, and M. S. Feld, "Measurement of angular distributions by use of low-coherence interferometry for light-scattering spectroscopy," Opt. Lett. 26, $322-324$ (2001).

4. D. Huang, E. A. Swanson, C. P. Lin, J. S. Schuman, W. G. Stinson, W. Chang, M. R. Hee, T. Flotte, K. Gregory, C. A. Puliafito, and J. G. Fujimoto, "Optical coherence tomography," Science 254, 1178-1181 (1991).

5. L. T. Perelman, V. Backman, M. Wallace, G. Zonios, R. Manohaan, A. Nusrat, S. Shields, M. Seiler, C. Lima, T. Hamamo, I. Itzkan, J. Van Dam, J. M. Crawford, and M. S. Feld, "Observation of periodic fine structure in reflectance from biological tissue: a new technique for measuring nuclear size distribution," Phys. Rev. Lett. 80, 627-630 (1998).

6. V. Backman, M. B. Wallace, L. T. Perelman, J. T. Arendt, R. Gurjar, M. G. Muller, O. Zhang, G. Zonios, E. Kline, T. McGillican, S. Shapshay, T. Valdez, K. Badizadegan, J. M. Crawford, M. Fitzmaurice, S. Kabani, H. S. Levin, M. Seiler, R. R. Dasari, I. Itzkan, J. Van Dam, and M. S. Feld, "Detection of preinvasive cancer cells," Nature 406, 35-36 (2000).

7. C. H. Yang, L. T. Perelman, A. Wax, R. R. Dasari, and M. S. Feld, "Feasibility of field-based light-scattering spectroscopy," J. Biomed. Opt. 5, 138-143 (2000).

8. A. Wax and J. E. Thomas, "Optical heterodyne imaging and Wigner phase-space distributions," Opt. Lett. 21, 14271429 (1996). 
9. A. Wax and J. E. Thomas, "Measurement of smoothed Wigner phase-space distributions for small-angle scattering in a turbid medium," J. Opt. Soc. Am. A 15, 1896-1908 (1998).

10. A. Wax, S. Bali, and J. E. Thomas, "Time-resolved phasespace distributions for light backscattered from a disordered medium," Phys. Rev. Lett. 85, 66 (2000).

11. It has been previously noted in Ref. 8 that the measured mean square heterodyne beat signal is related to the Wigner distribution of the signal field convoluted with that of the reference field. In the current experiments, the angular resolution is sufficiently small that we can regard our signal as the Wigner distribution of the backscattered field.

12. A. Wax, C. Yang, R. R. Dasari, and M. S. Feld, "Angular light scattering studies using low-coherence interferometry," in Coherence Domain Optical Methods in Biomedical Science and Clinical Applications V, V. V. Tuchin, J. A. Izatt, and J. G. Fujimoto, eds., Proc. SPIE 4251, 32-42 (2001). 